Pacific

Journal of

Mathematics

ADDITION FORMULAS FOR JACOBI THETA FUNCTIONS, DEDEKIND'S ETA FUNCTION, AND RAMANUJAN'S CONGRUENCES

\author{
ZHI-GUO LIU
}




\title{
ADDITION FORMULAS FOR JACOBI THETA FUNCTIONS, DEDEKIND'S ETA FUNCTION, AND RAMANUJAN'S CONGRUENCES
}

\author{
ZHI-GUO LIU
}

\begin{abstract}
Previously, we proved an addition formula for the Jacobi theta function, which allows us to recover many important classical theta function identities. Here, we use this addition formula to derive a curious theta function identity, which includes Jacobi's quartic identity and some other important theta function identities as special cases. We give new series expansions for $\eta^{2}(\tau), \eta^{6}(\tau), \eta^{8}(\tau)$, and $\eta^{10}(\tau)$, where $\eta(\tau)$ is Dedekind's eta function. The series expansions for $\eta^{6}(\tau)$ and $\eta^{10}(\tau)$ lead to simple proofs of Ramanujan's congruences $p(7 n+5) \equiv 0(\bmod 7)$ and $p(11 n+6) \equiv 0(\bmod 11)$, respectively.
\end{abstract}

\section{Introduction}

Throughout this paper we take $q=\exp (2 \pi i \tau)$, where $\tau$ has positive imaginary part. We first need to introduce the Jacobi theta functions.

Definition 1.1. The Jacobi theta functions $\theta_{k}$ for $k=1,2,3,4$ are defined as

$$
\begin{aligned}
& \theta_{1}(z \mid \tau)=2 \sum_{n=0}^{\infty}(-1)^{n} q^{(2 n+1)^{2} / 8} \sin (2 n+1) z \\
& \theta_{2}(z \mid \tau)=2 \sum_{n=0}^{\infty} q^{(2 n+1)^{2} / 8} \cos (2 n+1) z \\
& \theta_{3}(z \mid \tau)=1+2 \sum_{n=1}^{\infty} q^{n^{2} / 2} e^{2 n i z} \\
& \theta_{4}(z \mid \tau)=1+2 \sum_{n=1}^{\infty}(-1)^{n} q^{n^{2} / 2} e^{2 n i z}
\end{aligned}
$$

MSC2000: 33E05, 11F11, 11F20, 11F27.

Keywords: elliptic function, theta function, Dedekind's eta function, Ramanujan's congruence.

This work was supported by the National Science Foundation of China, PCSIRT, Innovation Program of Shanghai Municipal Education Commission, and Shanghai Leading Academic Discipline Project, project number B407. 
Using the theory of elliptic functions, we derived in [Liu 2007] the following general theta function identity.

Theorem 1.2. Let $h_{1}$ and $h_{2}$ be two entire functions of $z$ that satisfy the functional equations

$$
h_{i}(z \mid \tau)=-h_{i}(z+\pi \mid \tau)=-q^{3 / 2} e^{6 i z} h_{i}(z+\pi \tau \mid \tau) \quad \text { for } i=1,2 .
$$

Then there is a constant $C$ independent of $x$ and $y$ such that

$$
\begin{aligned}
& \left(h_{1}(x \mid \tau)-h_{1}(-x \mid \tau)\right)\left(h_{2}(y \mid \tau)-h_{2}(-y \mid \tau)\right) \\
-\left(h_{2}(x \mid \tau)-h_{2}(-x \mid \tau)\right)\left(h_{1}(y \mid \tau)-h_{1}(-y \mid \tau)\right) & \\
= & C \theta_{1}(x \mid \tau) \theta_{1}(y \mid \tau) \theta_{1}(x+y \mid \tau) \theta_{1}(x-y \mid \tau) .
\end{aligned}
$$

This identity was then used to derive many identities, including Ramanujan's cubic theta function identity, Winquist's identity, and the addition formula for Weierstrass's $\sigma$-function.

In this paper we will discuss additional applications of this identity. For brevity, we will use $\vartheta_{1}^{\prime}(\tau), \vartheta_{2}(\tau), \vartheta_{3}(\tau)$, and $\vartheta_{4}(\tau)$ to denote $\theta_{1}^{\prime}(0 \mid \tau), \theta_{2}(0 \mid \tau), \theta_{3}(0 \mid \tau)$, and $\theta_{4}(0 \mid \tau)$ respectively.

In Section 2, we shall use Theorem 1.2 to prove the following identity.

Theorem 1.3. Let $\theta_{1}, \theta_{2}, \theta_{3}$, and $\theta_{4}$ be the Jacobi theta functions. Then

$$
\begin{aligned}
& \theta_{1}(x+y \mid \tau) \theta_{1}(x-y \mid \tau) \theta_{2}(u+v \mid \tau) \theta_{2}(u-v \mid \tau)= \\
& \theta_{3}(y+u \mid \tau) \theta_{3}(y-u \mid \tau) \theta_{4}(x+v \mid \tau) \theta_{4}(x-v \mid \tau) \\
& -\theta_{3}(x+u \mid \tau) \theta_{3}(x-u \mid \tau) \theta_{4}(y+v \mid \tau) \theta_{4}(y-v \mid \tau) .
\end{aligned}
$$

This identity includes many well-known addition formulas for the Jacobi theta functions. In Section 3 we will derive this corollary from Theorem 1.3:

\section{Corollary 1.4.}

$$
2 \theta_{1}(x+y \mid \tau) \theta_{1}(x-y \mid \tau)=\theta_{3}(y \mid \tau / 2) \theta_{4}(x \mid \tau / 2)-\theta_{3}(x \mid \tau / 2) \theta_{4}(y \mid \tau / 2) .
$$

In Section 4, this identity will be used to derive the following remarkable fourterm theta function identity.

Theorem 1.5. Let $\theta_{1}, \theta_{2}, \theta_{3}$, and $\theta_{4}$ be the Jacobi theta functions. Then we have

$$
\begin{aligned}
2 \theta_{1}^{2}(x+y \mid \tau) \theta_{1}^{2}(x-y \mid \tau)= & \vartheta_{3}^{2}(\tau) \theta_{3}(2 x \mid \tau) \theta_{3}(2 y \mid \tau) \\
& -\vartheta_{2}^{2}(\tau) \theta_{2}(2 x \mid \tau) \theta_{2}(2 y \mid \tau) \\
& -\vartheta_{4}^{2}(\tau) \theta_{4}(2 x \mid \tau) \theta_{4}(2 y \mid \tau) .
\end{aligned}
$$


When $y=0$, this identity will reduce to the beautiful but little known identity [Enneper 1890, page 295, Equation (4)]

$$
2 \theta_{1}^{4}(x \mid \tau)=\vartheta_{3}^{3}(\tau) \theta_{3}(2 x \mid \tau)-\vartheta_{2}^{3}(\tau) \theta_{2}(2 x \mid \tau)-\vartheta_{4}^{3}(\tau) \theta_{4}(2 x \mid \tau) .
$$

With $\theta_{1}(0 \mid \tau)=0$, the $(x, y)=(0,0)$ case of Theorem 1.5 will give immediately the well-known Jacobi quartic identity

$$
\vartheta_{3}^{4}(\tau)=\vartheta_{2}^{4}(\tau)+\vartheta_{4}^{4}(\tau)
$$

Some applications of Theorem 1.5 to modular identities of degrees 3 and 5 are also discussed in Section 4.

For convenience, we introduce the $q$-shifted factorial $(a ; q)_{\infty}$ by

$$
(a ; q)_{\infty}:=\prod_{n=0}^{\infty}\left(1-a q^{n}\right) \text { for }|q|<1 .
$$

In this notation the well-known Dedekind eta function may be written as

$$
\eta(\tau)=q^{1 /(24)}(q ; q)_{\infty} .
$$

It is obvious that $\eta(\tau)$ is the same as Euler's product $(q ; q)_{\infty}$ except for an extra factor $q^{1 /(24)}$, and hence finding the series representation for $\eta^{r}(\tau)$ is equivalent to finding the series representation for $(q ; q)_{\infty}^{r}$, where $r$ is an integer.

With Corollary 1.4, in Sections 5, 6, 7, 8, we will give new series expansions of $\eta^{2 k}(\tau)$ for $k=1,3,4,5$, respectively. We will use the series expansions for $\eta^{6}(\tau)$ and $\eta^{10}(\tau)$ to give simple proofs of Ramanujan's congruences $p(7 n+5) \equiv$ $0(\bmod 5)$ and $p(11 n+6) \equiv 0(\bmod 11)$.

Theorem 1.6. $\quad(q ; q)_{\infty}^{2}=\frac{1}{2} \sum_{m, n=-\infty}^{\infty}\left((-1)^{n}-(-1)^{m}\right) q^{\left(3 m^{2}+3 n^{2}+4 m+1\right) / 4}$.

Theorem 1.7. $(q ; q)_{\infty}^{6}=\frac{1}{4} \sum_{m, n=-\infty}^{\infty}(-1)^{m}\left(n^{2}-m^{2}\right) q^{\left(m^{2}+n^{2}-1\right) / 4}$.

A corollary of Theorem 1.7 is the Ramanujan partition congruence modulo 7.

Corollary 1.8. Let $p(n)$ denote the number of unrestricted partitions of the positive integers $n$. Then $p(7 n+5) \equiv 0(\bmod 7)$.

Theorem 1.9. $(q ; q)_{\infty}^{8}=-\frac{1}{4} \sum_{m, n=-\infty}^{\infty} m^{2}(3 n+2)\left(1+(-1)^{m+n}\right) q^{\left(m^{2}+3 n^{2}+4 n\right) / 4}$.

\section{Theorem 1.10.}

$$
(q ; q)_{\infty}^{10}=\frac{1}{2} \sum_{m, n=-\infty}^{\infty}(3 m+2)(3 n+2)^{3}\left((-1)^{m}-(-1)^{n}\right) q^{\left(3 m^{2}+3 n^{2}+4 m+4 n+1\right) / 4} .
$$


A corollary of Theorem 1.10 is the Ramanujan partition congruence modulo 11 .

Corollary 1.11. Let $p(n)$ denote the number of unrestricted partitions of the positive integers $n$. Then $p(11 n+6) \equiv 0(\bmod 11)$.

Remark 1.12. Using Corollary 1.4 we can also derive for $\eta^{4}(\tau)$ the identity

$$
(q ; q)_{\infty}^{4}=\frac{1}{2} \sum_{m, n=-\infty}^{\infty}(-1)^{m+n}(2 n+1) q^{\left(n^{2}+3 m^{2}+n-m\right) / 2},
$$

which can be simply obtained by multiplying together Euler's pentagonal number identity for $(q ; q)_{\infty}$ and Jacobi's identity for $(q ; q)_{\infty}^{3}$, so we omit the details. This identity can be used to prove Ramanujan's congruence $p(5 n+4) \equiv 0(\bmod 5)$; see for example [Hardy and Wright 1979, pages 287-289].

In this paper we also need the infinite product representations of theta functions. We recall the Jacobi triple product identity (see for example [Andrews et al. 1999, page 497; Berndt 1991, page 35; Berndt 2006, page 10; Hardy and Wright 1979, page 282; Kongsiriwong and Liu 2003])

$$
(q ; q)_{\infty}(z ; q)_{\infty}(q / z ; q)_{\infty}=\sum_{n=-\infty}^{\infty}(-1)^{n} q^{n(n-1) / 2} z^{n} .
$$

Replacing $z$ with $e^{2 i z}$ in the Jacobi triple product identity will give

$$
\theta_{1}(z \mid \tau)=2 q^{1 / 8}(\sin z)(q ; q)_{\infty}\left(q e^{2 i z} ; q\right)_{\infty}\left(q e^{-2 i z} ; q\right)_{\infty} .
$$

From the definitions of theta functions, by direct computations, we readily find that

$$
\begin{aligned}
& \theta_{2}(z \mid \tau)=\theta_{1}(z+\pi / 2 \mid \tau), \\
& \theta_{3}(z \mid \tau)=q^{1 / 8} e^{i z} \theta_{1}(z+(\pi+\pi \tau) / 2 \mid \tau), \\
& \theta_{4}(z \mid \tau)=-i q^{1 / 8} e^{i z} \theta_{1}(z+(\pi \tau) / 2 \mid \tau) .
\end{aligned}
$$

Combining (1-2) and (1-3) gives the infinite product representations of $\theta_{2}, \theta_{3}$, and $\theta_{4}$ :

$$
\begin{aligned}
& \theta_{2}(z \mid \tau)=2 q^{1 / 8}(\cos z)(q ; q)_{\infty}\left(-q e^{2 i z} ; q\right)_{\infty}\left(-q e^{-2 i z} ; q\right)_{\infty}, \\
& \theta_{3}(z \mid \tau)=(q ; q)_{\infty}\left(-q^{1 / 2} e^{2 i z} ; q\right)_{\infty}\left(-q^{1 / 2} e^{-2 i z} ; q\right)_{\infty}, \\
& \theta_{4}(z \mid \tau)=(q ; q)_{\infty}\left(q^{1 / 2} e^{2 i z} ; q\right)_{\infty}\left(q^{1 / 2} e^{-2 i z} ; q\right)_{\infty} .
\end{aligned}
$$

Differentiating (1-2) with respect to $z$ and then putting $z=0$ will yield

$$
\vartheta_{1}^{\prime}(\tau)=2 q^{1 / 8}(q ; q)_{\infty}^{3}=2 \eta^{3}(\tau)
$$




\section{The proof of Theorem 1.3}

Our main aim of this section is to prove Theorem 1.3 using Theorem 1.2.

Proof. From the definitions of theta functions in Definition 1.1, we readily find that

$$
\begin{aligned}
& \theta_{1}(z \mid \tau)=-\theta_{1}(z+\pi \mid \tau)=-q^{1 / 2} e^{2 i z} \theta_{1}(z+\pi \tau \mid \tau), \\
& \theta_{3}(z \mid \tau)=\theta_{3}(z+\pi \mid \tau)=q^{1 / 2} e^{2 i z} \theta_{3}(z+\pi \tau \mid \tau), \\
& \theta_{4}(z \mid \tau)=\theta_{4}(z+\pi \mid \tau)=-q^{1 / 2} e^{2 i z} \theta_{4}(z+\pi \tau \mid \tau) .
\end{aligned}
$$

With these functional equations and by direct computations, we can easily verify that

$$
\theta_{1}(z \mid \tau) \theta_{3}(z-v \mid \tau) \theta_{3}(z+v \mid \tau) \quad \text { and } \quad \theta_{1}(z \mid \tau) \theta_{4}(z-u \mid \tau) \theta_{4}(z+u \mid \tau)
$$

satisfy all the conditions of Theorem 1.2. Thus we can choose $h_{1}$ and $h_{2}$ as

$$
\begin{aligned}
& h_{1}(z \mid \tau)=\frac{1}{2} \theta_{1}(z \mid \tau) \theta_{3}(z-v \mid \tau) \theta_{3}(z+v \mid \tau), \\
& h_{2}(z \mid \tau)=\frac{1}{2} \theta_{1}(z \mid \tau) \theta_{4}(z-u \mid \tau) \theta_{4}(z+u \mid \tau)
\end{aligned}
$$

in Theorem 1.2 and then cancel out the common factor $\theta_{1}(x \mid \tau) \theta_{1}(y \mid \tau)$ in the resulting equation to obtain

$$
\begin{array}{r}
C \theta_{1}(x+y \mid \tau) \theta_{1}(x-y \mid \tau)= \\
\theta_{3}(y+u \mid \tau) \theta_{3}(y-u \mid \tau) \theta_{4}(x+v \mid \tau) \theta_{4}(x-v \mid \tau) \\
-\theta_{3}(x+u \mid \tau) \theta_{3}(x-u \mid \tau) \theta_{4}(y+v \mid \tau) \theta_{4}(y-v \mid \tau) .
\end{array}
$$

Putting $x=v+(\pi \tau) / 2$ in the this equation and then using the fact $\theta_{4}((\pi \tau) / 2 \mid \tau)=0$ in the resulting equation, we find that

$$
C \theta_{4}(y+u \mid \tau) \theta_{4}(y-u \mid \tau)=\theta_{4}(y+u \mid \tau) \theta_{4}(y-u \mid \tau) \theta_{2}(u+v \mid \tau) \theta_{2}(u-v \mid \tau) .
$$

It follows that $C=\theta_{2}(u+v \mid \tau) \theta_{2}(u-v \mid \tau)$. Substituting this into (2-1), we arrive at Theorem 1.3.

Theorem 1.3 contains some interesting cases. If we let $(u, v)$ equal

$$
(0,0), \quad((\pi \tau) / 2,0), \quad((\pi+\pi \tau) / 2,0), \quad((\pi \tau) / 2, \quad(\pi \tau) / 2), \quad(0,(\pi \tau) / 2)
$$


we find, respectively, that

$$
\begin{aligned}
& \vartheta_{2}^{2}(\tau) \theta_{1}(x-y \mid \tau) \theta_{1}(x+y \mid \tau)=\theta_{3}^{2}(y \mid \tau) \theta_{4}^{2}(x \mid \tau)-\theta_{4}^{2}(y \mid \tau) \theta_{3}^{2}(x \mid \tau), \\
& \vartheta_{3}^{2}(\tau) \theta_{1}(x-y \mid \tau) \theta_{1}(x+y \mid \tau)=\theta_{2}^{2}(y \mid \tau) \theta_{4}^{2}(x \mid \tau)-\theta_{4}^{2}(y \mid \tau) \theta_{2}^{2}(x \mid \tau), \\
& \vartheta_{4}^{2}(\tau) \theta_{1}(x-y \mid \tau) \theta_{1}(x+y \mid \tau)=\theta_{1}^{2}(x \mid \tau) \theta_{4}^{2}(y \mid \tau)-\theta_{1}^{2}(y \mid \tau) \theta_{4}^{2}(x \mid \tau), \\
& \vartheta_{2}^{2}(\tau) \theta_{1}(x-y \mid \tau) \theta_{1}(x+y \mid \tau)=\theta_{1}^{2}(x \mid \tau) \theta_{2}^{2}(y \mid \tau)-\theta_{1}^{2}(y \mid \tau) \theta_{2}^{2}(x \mid \tau), \\
& \vartheta_{3}^{2}(\tau) \theta_{1}(x-y \mid \tau) \theta_{1}(x+y \mid \tau)=\theta_{1}^{2}(x \mid \tau) \theta_{3}^{2}(y \mid \tau)-\theta_{1}^{2}(y \mid \tau) \theta_{3}^{2}(x \mid \tau) .
\end{aligned}
$$

The identities of (2-2) are usually called the addition formulas for the theta functions and were known to Jacobi. See [Enneper 1890, pages 107-108] for more identities of this type.

\section{The proof of Corollary 1.4 and its dual form}

In this section we will use Theorem 1.3 to prove Corollary 1.4 and its dual form

$$
\theta_{1}(x+y \mid \tau) \theta_{1}(x-y \mid \tau)=\theta_{2}(2 y \mid \tau) \theta_{3}(2 x \mid \tau)-\theta_{3}(2 y \mid \tau) \theta_{2}(2 x \mid 2 \tau) .
$$

Proof. Appealing to the infinite product representations of theta functions and using direct computation, we find that

$$
\begin{aligned}
2 \theta_{4}(z+(\pi \tau) / 4 \mid \tau) \theta_{4}(z-(\pi \tau) / 4 \mid \tau) & =q^{-1 /(16)} \vartheta_{2}(\tau / 2) \theta_{4}(z \mid \tau / 2), \\
2 \theta_{3}(z+(\pi \tau) / 4 \mid \tau) \theta_{3}(z-(\pi \tau) / 4 \mid \tau) & =q^{-1 /(16)} \vartheta_{2}(\tau / 2) \theta_{3}(z \mid \tau / 2), \\
2 \vartheta_{2}(\tau) \theta_{2}((\pi \tau) / 2 \mid \tau) & =q^{-1 / 8} \vartheta_{2}^{2}(\tau / 2) .
\end{aligned}
$$

Setting $u=v=(\pi \tau) / 4$ in Theorem 1.3, using (3-2), and canceling out the common factor $q^{-1 / 8} \theta_{2}^{2}(0 \mid \tau / 2)$, we obtain Corollary 1.4.

Now we will use Corollary 1.4 to derive (3-1). First, by replacing $(\tau, x, y)$ by $(-1 / \tau, x / \tau, y / \tau)$ in Corollary 1.4, we have

$$
\begin{aligned}
\theta_{1}\left(\frac{x+y}{\tau} \mid-\frac{1}{\tau}\right) \theta_{1}\left(\frac{x-y}{\tau} \mid-\frac{1}{\tau}\right) \\
\quad=\theta_{2}\left(\frac{y}{\tau} \mid-\frac{1}{2 \tau}\right) \theta_{3}\left(\frac{x}{\tau} \mid-\frac{1}{2 \tau}\right)-\theta_{3}\left(\frac{y}{\tau} \mid-\frac{1}{2 \tau}\right) \theta_{2}\left(\frac{x}{\tau} \mid-\frac{1}{2 \tau}\right)
\end{aligned}
$$

We apply the imaginary transformation formulas

$$
\begin{aligned}
& \theta_{1}\left(\frac{z}{\tau} \mid-\frac{1}{\tau}\right)=-i \sqrt{-i \tau} \exp \left(\left(i z^{2}\right) /(\pi \tau)\right) \theta_{1}(z \mid \tau), \\
& \theta_{2}\left(\frac{z}{\tau} \mid-\frac{1}{\tau}\right)=\sqrt{-i \tau} \exp \left(\left(i z^{2}\right) /(\pi \tau)\right) \theta_{4}(z \mid \tau) \\
& \theta_{3}\left(\frac{z}{\tau} \mid-\frac{1}{\tau}\right)=\sqrt{-i \tau} \exp \left(\left(i z^{2}\right) /(\pi \tau)\right) \theta_{3}(z \mid \tau) \\
& \theta_{4}\left(\frac{z}{\tau} \mid-\frac{1}{\tau}\right)=\sqrt{-i \tau} \exp \left(\left(i z^{2}\right) /(\pi \tau)\right) \theta_{2}(z \mid \tau)
\end{aligned}
$$


in (3-3) and then cancel out the common factors to obtain (3-1). Thus (3-1) and Corollary 1.4 are equivalent under the imaginary transformations. This completes the proofs of Corollary 1.4 and its dual form.

Remark 3.1. Replacing $x$ by $x+\pi / 2$ in (3-1), we immediately find that

$$
\theta_{2}(x+y \mid \tau) \theta_{2}(x-y \mid \tau)=\theta_{2}(2 y \mid 2 \tau) \theta_{3}(2 x \mid 2 \tau)+\theta_{3}(2 y \mid \tau) \theta_{2}(2 x \mid 2 \tau),
$$

which is the same as [Enneper 1890, page 140, Equation (16)] and was known to Jacobi. So (3-1) is just a variant of Jacobi's identity (3-4). Ewell [1995] rediscovered (3-1) and called it a sextuple product identity. Corollary 1.4 first appeared in [Shen 1994, page 327, Equation (1.3d)] in a different form, and we may call it the Jacobi-Shen identity.

\section{The proof of Theorem 1.5 and its applications}

The proof of Theorem 1.5. Taking $\tau$ to $2 \tau$ in Corollary 1.4 and then replacing $x$ by $x+\pi / 2$ and $x+(\pi+2 \pi \tau) / 2$ in the resulting equations, we find respectively that

$$
\begin{aligned}
& 2 \theta_{2}(x+y \mid 2 \tau) \theta_{2}(x-y \mid 2 \tau)=\theta_{3}(x \mid \tau) \theta_{3}(y \mid \tau)-\theta_{4}(x \mid \tau) \theta_{4}(y \mid \tau), \\
& 2 \theta_{3}(x+y \mid 2 \tau) \theta_{3}(x-y \mid 2 \tau)=\theta_{3}(x \mid \tau) \theta_{3}(y \mid \tau)+\theta_{4}(x \mid \tau) \theta_{4}(y \mid \tau) .
\end{aligned}
$$

Taking $y=0$ in these two equations and then replacing $x$ by $2 x$, we have

$$
\begin{aligned}
& 2 \theta_{2}^{2}(2 x \mid 2 \tau)=\vartheta_{3}(\tau) \theta_{3}(2 x \mid \tau)-\vartheta_{4}(\tau) \theta_{4}(2 x \mid \tau), \\
& 2 \theta_{3}^{2}(2 x \mid 2 \tau)=\vartheta_{3}(\tau) \theta_{3}(2 x \mid \tau)+\vartheta_{4}(\tau) \theta_{4}(2 x \mid \tau) .
\end{aligned}
$$

Replacing $x$ by $y$ in (4-2) and then multiplying the resulting equation with (4-1), we find that

$$
\begin{aligned}
& 4 \theta_{2}^{2}(2 x \mid 2 \tau) \theta_{3}^{2}(2 y \mid 2 \tau)=\vartheta_{3}^{2}(\tau) \theta_{3}(2 x \mid \tau) \theta_{3}(2 y \mid \tau)-\vartheta_{4}^{2}(\tau) \theta_{4}(2 x \mid \tau) \theta_{4}(2 y \mid \tau) \\
& +\vartheta_{3}(\tau) \vartheta_{4}(\tau) \theta_{3}(2 x \mid \tau) \theta_{4}(2 y \mid \tau)-\vartheta_{3}(\tau) \vartheta_{4}(\tau) \theta_{4}(2 x \mid \tau) \theta_{3}(2 y \mid \tau) .
\end{aligned}
$$

If we interchange $x$ and $y$ in the above equation, then we immediately find that

$$
\begin{aligned}
& 4 \theta_{2}^{2}(2 y \mid 2 \tau) \theta_{3}^{2}(2 x \mid 2 \tau)=\vartheta_{3}^{2}(\tau) \theta_{3}(2 x \mid \tau) \theta_{3}(2 y \mid \tau)-\vartheta_{4}^{2}(\tau) \theta_{4}(2 x \mid \tau) \theta_{4}(2 y \mid \tau) \\
& +\vartheta_{3}(\tau) \vartheta_{4}(\tau) \theta_{3}(2 y \mid \tau) \theta_{4}(2 x \mid \tau)-\vartheta_{3}(\tau) \vartheta_{4}(\tau) \theta_{4}(2 y \mid \tau) \theta_{3}(2 x \mid \tau) .
\end{aligned}
$$

Adding the above two equations together and simplifying, we conclude that

$$
\begin{aligned}
& 2 \theta_{2}^{2}(2 x \mid 2 \tau) \theta_{3}^{2}(2 y \mid 2 \tau)+2 \theta_{2}^{2}(2 y \mid 2 \tau) \theta_{3}^{2}(2 x \mid 2 \tau) \\
& =\vartheta_{3}^{2}(\tau) \theta_{3}(2 x \mid \tau) \theta_{3}(2 y \mid \tau)-\vartheta_{4}^{2}(\tau) \theta_{4}(2 x \mid \tau) \theta_{4}(2 y \mid \tau)
\end{aligned}
$$

Using the infinite product representations of the theta functions, we readily find

$$
2 \theta_{2}(2 z \mid 2 \tau) \theta_{3}(2 z \mid 2 \tau)=\vartheta_{2}(\tau) \theta_{2}(2 z \mid \tau) .
$$


Squaring both sides of (3-1) and then using (4-4) in the resulting equation, we find that

$$
\begin{aligned}
\theta_{1}^{2}(x+y \mid \tau) \theta_{1}^{2}(x-y \mid \tau)=\theta_{2}^{2}(2 y \mid 2 \tau) \theta_{3}^{2}(2 x \mid 2 \tau)+ & \theta_{2}^{2}(2 x \mid 2 \tau) \theta_{3}^{2}(2 y \mid 2 \tau) \\
& -\frac{1}{2} \vartheta_{2}^{2}(\tau) \theta_{2}(2 x \mid \tau) \theta_{2}(2 y \mid \tau) .
\end{aligned}
$$

Combining this identity with (4-3), we arrive at Theorem 1.5.

Some modular identities of degrees 3 and 5. By taking $y=0$ and $x=\pi / 3$ in Theorem 1.5, we immediately have

$$
2 \theta_{1}^{4}(\pi / 3 \mid \tau)=\vartheta_{2}^{3}(\tau) \theta_{2}(\pi / 3 \mid \tau)+\vartheta_{3}^{3}(\tau) \theta_{3}(\pi / 3 \mid \tau)-\vartheta_{4}^{3}(\tau) \theta_{4}(\pi / 3 \mid \tau) .
$$

Using the infinite product representations of theta functions, we find that

$$
\begin{aligned}
& \theta_{1}(\pi / 3 \mid \tau)=\sqrt{3} q^{1 / 8}\left(q^{3} ; q^{3}\right)_{\infty}, \\
& \theta_{j}(\pi / 3 \mid \tau)=\sqrt{\frac{(q ; q)_{\infty}^{3}}{\left(q^{3} ; q^{3}\right)_{\infty}} \times \frac{\vartheta_{j}(3 \tau)}{\vartheta_{j}(\tau)}} \text { for } j=2,3,4 .
\end{aligned}
$$

Substituting these equations into (4-5) and simplifying, we can deduce that

$$
\sqrt{\vartheta_{2}^{5}(\tau) \vartheta_{2}(3 \tau)}+\sqrt{\vartheta_{3}^{5}(\tau) \vartheta_{3}(3 \tau)}-\sqrt{\vartheta_{4}^{5}(\tau) \vartheta_{4}(3 \tau)}=9 \sqrt{\vartheta_{1}^{\prime}(3 \tau)^{3} / \vartheta_{1}^{\prime}(\tau)} .
$$

Applying the imaginary transformation to this identity, we conclude that

$$
\sqrt{\vartheta_{3}^{5}(3 \tau) \vartheta_{3}(\tau)}-\sqrt{\vartheta_{2}^{5}(3 \tau) \vartheta_{2}(\tau)}+\sqrt{\vartheta_{4}^{5}(3 \tau) \vartheta_{4}(\tau)}=\sqrt{\vartheta_{1}^{\prime}(\tau)^{3} / \vartheta_{1}^{\prime}(3 \tau)} .
$$

The above two identities are equivalent, respectively, to the two modular equations

$$
\begin{aligned}
& \left(\alpha^{5} \beta\right)^{1 / 8}-\left((1-\alpha)^{5}(1-\beta)\right)^{1 / 8}+1=\frac{9}{m^{2}}\left(\frac{\beta^{3}(1-\beta)^{3}}{\alpha(1-\alpha)}\right)^{1 / 8}, \\
& 1-\left(\alpha \beta^{5}\right)^{1 / 8}+\left((1-\alpha)(1-\beta)^{5}\right)^{1 / 8}=m^{2}\left(\frac{\alpha^{3}(1-\alpha)^{3}}{\beta(1-\beta)}\right)^{1 / 8},
\end{aligned}
$$

where $\alpha=\vartheta_{2}^{4}(\tau) / \vartheta_{3}^{4}(\tau), \quad \beta=\vartheta_{2}^{4}(3 \tau) / \vartheta_{3}^{4}(3 \tau)$ and $m=\vartheta_{3}^{2}(\tau) / \vartheta_{3}^{2}(3 \tau)$.

Using the infinite product representations of theta functions, we easily find that

$$
\begin{aligned}
& \theta_{1}(\pi / 5 \mid \tau) \theta_{1}(2 \pi / 5 \mid \tau)=\sqrt{5} q^{1 / 4}(q ; q)_{\infty}\left(q^{5} ; q^{5}\right)_{\infty}, \\
& \theta_{j}(\pi / 5 \mid \tau) \theta_{j}(2 \pi / 5 \mid \tau)=\sqrt{\frac{(q ; q)_{\infty}^{5}}{\left(q^{5} ; q^{5}\right)_{\infty}} \times \frac{\vartheta_{j}(5 \tau)}{\vartheta_{j}(\tau)}} \text { for } j=2,3,4 .
\end{aligned}
$$

Setting $x=\pi / 5$ and $y=(2 \pi) / 5$ in Theorem 1.5 and simplifying, we find

$$
\begin{aligned}
& 2 \theta_{1}^{2}(\pi / 5 \mid \tau) \theta_{1}^{2}(2 \pi / 5 \mid \tau)=\vartheta_{2}^{2}(\tau) \theta_{2}(\pi / 5 \mid \tau) \theta_{2}(2 \pi / 5 \mid \tau) \\
& +\vartheta_{3}^{2}(\tau) \theta_{3}(\pi / 5 \mid \tau) \theta_{3}(2 \pi / 5 \mid \tau)-\vartheta_{4}^{2}(\tau) \theta_{4}(\pi / 5 \mid \tau) \theta_{4}(2 \pi / 5 \mid \tau) .
\end{aligned}
$$


Substituting the equations of (4-6) into the above equations and simplifying, we find that [Shen 1995]

$$
\sqrt{\vartheta_{2}^{3}(\tau) \vartheta_{2}(5 \tau)}+\sqrt{\vartheta_{3}^{3}(\tau) \vartheta_{3}(5 \tau)}-\sqrt{\vartheta_{4}^{3}(\tau) \vartheta_{4}(5 \tau)}=10 \sqrt{\eta^{5}(5 \tau) / \eta(\tau)} .
$$

Applying the imaginary transformation to this identity, we find that [Shen 1995]

$$
\sqrt{\vartheta_{3}(\tau) \vartheta_{3}^{3}(5 \tau)}-\sqrt{\vartheta_{2}(\tau) \vartheta_{2}^{3}(5 \tau)}+\sqrt{\vartheta_{4}(\tau) \vartheta_{4}^{3}(5 \tau)}=2 \sqrt{\eta^{5}(\tau) / \eta(5 \tau)} .
$$

The two identities above are equivalent, respectively, to the two modular equations

$$
\begin{aligned}
& 1+\left(\alpha^{3} \beta\right)^{1 / 8}-\left((1-\alpha)^{3}(1-\beta)\right)^{1 / 8}=\frac{5 \sqrt[3]{2}}{m}\left(\frac{\beta^{5}(1-\beta)^{5}}{\alpha(1-\alpha)}\right)^{1 / 24} \\
& 1-\left(\alpha \beta^{3}\right)^{1 / 8}+\left((1-\alpha)(1-\beta)^{3}\right)^{1 / 8}=\sqrt[3]{2} m\left(\frac{\alpha^{5}(1-\alpha)^{5}}{\beta(1-\beta)}\right)^{1 / 24}
\end{aligned}
$$

where $\alpha=\vartheta_{2}^{4}(\tau) / \vartheta_{3}^{4}(\tau), \quad \beta=\vartheta_{2}^{4}(5 \tau) / \vartheta_{3}^{4}(5 \tau)$, and $m=\vartheta_{3}^{2}(\tau) / \vartheta_{3}^{2}(5 \tau)$.

\section{The proof of Theorem 1.6}

Proof. Replacing $\tau$ by $3 \tau$ in Corollary 1.4 and then setting $(x, y)=(\pi \tau, 0)$ in the resulting equation, we deduce that

$$
2 \theta_{1}^{2}(\pi \tau \mid 3 \tau)=\vartheta_{3}(3 \tau / 2) \theta_{4}(\pi \tau \mid \tau / 2)-\vartheta_{4}(3 \tau / 2) \theta_{3}(\pi \tau \mid \tau / 2) .
$$

Appealing to the infinite product representation of $\theta_{1}$, we find easily that

$$
\theta_{1}(\pi \tau \mid 3 \tau)=i q^{-1 / 8}(q ; q)_{\infty} .
$$

Using the series representations of theta functions, we immediately find that

$$
\begin{array}{ll}
\vartheta_{3}(3 \tau / 2)=\sum_{n=-\infty}^{\infty} q^{3 n^{2} / 4}, & \theta_{4}(\pi \tau \mid \tau / 2)=\sum_{n=-\infty}^{\infty}(-1)^{n} q^{\left(3 n^{2}+4 n\right) / 4}, \\
\vartheta_{4}(3 \tau / 2)=\sum_{n=-\infty}^{\infty}(-1)^{n} q^{3 n^{2} / 4}, & \theta_{3}(\pi \tau \mid \tau / 2)=\sum_{n=-\infty}^{\infty} q^{\left(3 n^{2}+4 n\right) / 4},
\end{array}
$$

Substituting the five equations above into (5-1) and using a direct computation, we arrive at Theorem 1.6.

There are several different series representations for $\eta^{2}(\tau)$ in the literature. In a famous paper, L. J. Rogers [1894] first proved the identity

$$
(q ; q)_{\infty}^{2}=\sum_{\substack{m, n=-\infty \\ n \geq 2|m|}}^{\infty}(-1)^{m+n} q^{n(n+1) / 2-m(3 m-1) / 2} .
$$


In [1959, pages 418-427], Hecke rediscovered this identity. Andrews [1984; 1986] and Kac and Peterson [1980] reproved this identity recently.

Liu [2002] proved the identity

$$
(q ; q)_{\infty}^{2}=\sum_{n=0}^{\infty} \sum_{j=-n}^{n}(-1)^{j}\left(1-q^{2 n+1}\right) q^{2 n^{2}+n-j(3 j+1) / 2}
$$

using a general $q$-series expansion formula.

Ewell [1982] and Shen [1999] respectively found these two formulas for $\eta^{2}(\tau)$ :

$$
\begin{aligned}
& (q ; q)_{\infty}^{2}=\sum_{m, n=-\infty}^{\infty}\left(q^{3 m^{2}+3 n^{2}+n}-q^{3 m^{2}+3 n^{2}+3 m+2 n+1}\right), \\
& (q ; q)_{\infty}^{2}=\sum_{m, n=-\infty}^{\infty}(-1)^{m} q^{m^{2}+n^{2}+m n+n / 2}
\end{aligned}
$$

\section{The proofs of Theorem 1.7 and Corollary 1.8}

Proof. Differentiating both sides of Corollary 1.4 with respect to $x$ twice and then putting $x=y=0$ in the resulting equation and noting that $\theta_{1}(0 \mid \tau)=\theta_{1}^{\prime \prime}(0 \mid \tau)=0$, we conclude that

$$
4 \vartheta_{1}^{\prime}(\tau)^{2}=\vartheta_{3}(\tau / 2) \theta_{4}^{\prime \prime}(0 \mid \tau / 2)-\vartheta_{4}(\tau / 2) \theta_{3}^{\prime \prime}(0 \mid \tau / 2)
$$

Substituting $4 \vartheta_{1}^{\prime}(\tau)^{2}=16 q^{1 / 4}(q ; q)_{\infty}^{6}$ and the series expansions of $\theta_{3}$ and $\theta_{4}$ into this equation, we immediately arrive at the equation of Theorem 1.7.

Next we will prove Corollary 1.8 with the help of Theorem 1.7. If we write

$$
(q ; q)_{\infty}^{6}=\sum_{n=0}^{\infty} a(n) q^{n}
$$

then equating coefficients of $q^{n}$ for $n \geq 1$ on both sides of Theorem 1.7, we find

$$
a(n)=\frac{1}{4} \sum_{\substack{u, v=-\infty \\ u^{2}+v^{2}-1=4 n}}^{\infty}(-1)^{u}\left(v^{2}-u^{2}\right) .
$$

If $n \equiv 5(\bmod 7)$, then $u^{2}+v^{2} \equiv 0(\bmod 7)$. By examining all cases modulo 7, we find that both $u, v \equiv 0(\bmod 7)$. It follows from $(6-2)$ that

$$
a(7 n+5) \equiv 0\left(\bmod 7^{2}\right) .
$$


Now from (6-1), we have

$$
\sum_{n=0}^{\infty} p(n) q^{n}=\frac{1}{(q ; q)_{\infty}}=\frac{(q ; q)_{\infty}^{6}}{(q ; q)_{\infty}^{7}} \equiv \frac{(q ; q)_{\infty}^{6}}{\left(q^{7} ; q^{7}\right)_{\infty}}=\frac{\sum_{n=0}^{\infty} a(n) q^{n}}{\left(q^{7} ; q^{7}\right)_{\infty}}(\bmod 7) .
$$

Extracting those terms with indices of the form $7 n+5$ and employing (6-3), we conclude that

$$
\sum_{n=0}^{\infty} p(7 n+5) q^{7 n+5} \equiv \frac{\sum_{n=0}^{\infty} a(7 n+5) q^{7 n+5}}{\left(q^{7} ; q^{7}\right)_{\infty}} \equiv 0(\bmod 7) .
$$

Thus we have $p(7 n+5) \equiv 0(\bmod 7)$. This proves Corollary 1.8 .

Schoeneberg [1953] gave a beautiful formula for $\eta^{6}(\tau)$ :

$$
(q ; q)_{\infty}^{6}=\sum_{m, n=-\infty}^{\infty} \operatorname{Re}(m+2 n i)^{2} q^{\left(m^{2}+4 n^{2}-1\right) / 4} .
$$

Hirschhorn [1983] used his septuple product identity to give a series representation for $\eta^{6}(\tau)$, which he then used to prove that $p(7 n+5) \equiv 0(\bmod 7)$.

Ewell [1982] provided a series representation for $\eta^{6}(\tau)$ from a theta function identity of Gauss, which let to an alternative proof of $p(7 n+5) \equiv 0(\bmod 7)$.

\section{The proof of Theorem 1.9}

Proof. Differentiating both sides of Corollary 1.4 with respect to $x$ and then setting $y=x$, we find that

$$
2 \vartheta_{1}^{\prime}(\tau) \theta_{1}(2 x \mid \tau)=\theta_{3}(x \mid \tau / 2) \theta_{4}^{\prime}(x \mid \tau / 2)-\theta_{3}^{\prime}(x \mid \tau / 2) \theta_{4}(x \mid \tau / 2) .
$$

Now we introduce two theta functions $Q_{1}(x \mid \tau)$ and $Q_{2}(x \mid \tau)$ by

$$
\begin{aligned}
& Q_{1}(x \mid \tau)=(q ; q)_{\infty}^{-1} \theta_{1}(2 x \mid \tau) \theta_{3}(x \mid \tau / 2), \\
& Q_{2}(x \mid \tau)=(q ; q)_{\infty}^{-1} \theta_{1}(2 x \mid \tau) \theta_{4}(x \mid \tau / 2) .
\end{aligned}
$$

Using the infinite product representations of theta functions and a direct computation, we easily find that

$$
\theta_{1}(x \mid \tau) \theta_{2}(x \mid \tau)=\vartheta_{4}(2 \tau) \theta_{1}(2 x \mid 2 \tau) .
$$

Combining this equation with [Shen 1999, Equations (a) and (b)], we deduce that

$$
\begin{aligned}
& Q_{1}(x \mid \tau)=2 q^{3 / 8} \sum_{n=-\infty}^{\infty}(-1)^{n} q^{\left(3 n^{2}+4 n\right) / 4} \sin (6 n+4) x, \\
& Q_{2}(x \mid \tau)=-2 q^{3 / 8} \sum_{n=-\infty}^{\infty} q^{\left(3 n^{2}+4 n\right) / 4} \sin (6 n+4) x
\end{aligned}
$$


We multiply both sides of (7-1) by $(q ; q)_{\infty}^{-1} \theta_{1}(2 x \mid \tau)$ and obtain the identity

$$
2(q ; q)_{\infty}^{-1} \vartheta_{1}^{\prime}(\tau) \theta_{1}(2 x \mid \tau)=Q_{1}(x \mid \tau) \theta_{4}^{\prime}(x \mid \tau / 2)-Q_{2}(x \mid \tau) \theta_{3}^{\prime}(x \mid \tau / 2) .
$$

Dividing this equation by $x^{2}$ and then letting $x \rightarrow 0$, we conclude that

$$
8(q ; q)_{\infty}^{-1} \vartheta_{1}^{\prime}(\tau)^{3}=Q_{1}^{\prime}(0 \mid \tau) \theta_{4}^{\prime \prime}(0 \mid \tau / 2)-Q_{2}^{\prime}(0 \mid \tau) \theta_{3}^{\prime \prime}(0 \mid \tau / 2) .
$$

Substituting $\vartheta_{1}^{\prime}(\tau)=2 \eta^{3}(\tau)$, (7-2), and the series expansion of $\theta_{3}$ and $\theta_{4}$ into (7-3) and simplifying, we arrive at Theorem 1.9.

Klein and Fricke [1890] (see also [Chan et al. 2007]) derived for $\eta^{8}(\tau)$ that

$$
(q ; q)_{\infty}^{8}=\frac{1}{2} \sum_{\substack{\alpha \equiv 1(\bmod 3) \\ \beta \equiv 1(\bmod 3)}}(\alpha+\beta)(2 \alpha-\beta)(2 \beta-\alpha) q^{\left(\alpha^{2}+\beta^{2}-\alpha \beta-1\right) / 3} .
$$

Winquist [1969] (see also [Chan et al. 2007]) stated without proof that

$$
(q ; q)_{\infty}^{8}=\frac{1}{2} \sum_{\substack{\alpha \equiv 1(\bmod 3) \\ \alpha+\beta \equiv 0(\bmod 3)}} \alpha \beta^{2} q^{\left(\alpha^{2}+3 \beta^{2}-4\right) / 12} .
$$

Schoeneberg [1953, Equation (11)] found a curious formula for $\eta^{8}(\tau)$ :

$$
(q ; q)_{\infty}^{8}=\frac{1}{6} \sum_{\mu \in \mathbb{Z}[\exp (2 \pi i / 3)]} \chi(\mu) \mu^{3} q^{\left(|\mu|^{2}-1\right) / 3},
$$

where

$$
\chi(\mu)=\left\{\begin{aligned}
1 & \text { if } \mu \equiv 1(\bmod \sqrt{-3}) \\
-1 & \text { if } \mu \equiv-1(\bmod \sqrt{-3}) .
\end{aligned}\right.
$$

\section{The proofs of Theorem 1.10 and Corollary 1.11}

Proof. Let $Q_{1}$ and $Q_{2}$ be as in (7-2). Multiplying both sides of Corollary 1.4 by $(q ; q)_{\infty}^{-2} \theta_{1}(2 x \mid \tau) \theta_{1}(2 y \mid \tau)$, we find that

$$
\begin{aligned}
2(q ; q)_{\infty}^{-2} \theta_{1}(2 x \mid \tau) \theta_{1}(2 y \mid \tau) \theta_{1}(x+y \mid \tau) & \theta_{1}(x-y \mid \tau) \\
& =Q_{1}(y \mid \tau) Q_{2}(x \mid \tau)-Q_{1}(x \mid \tau) Q_{2}(y \mid \tau) .
\end{aligned}
$$

Differentiating this equation with respect to $x$ and then setting $y=x$, we find that

$$
2(q ; q)_{\infty}^{-2} \vartheta_{1}^{\prime}(\tau) \theta_{1}^{3}(2 x \mid \tau)=Q_{1}(x \mid \tau) Q_{2}^{\prime}(x \mid \tau)-Q_{1}^{\prime}(x \mid \tau) Q_{2}(x \mid \tau) .
$$

Dividing this equation by $x^{3}$ and then letting $x \rightarrow 0$, we arrive at

$$
16(q ; q)_{\infty}^{-2} \vartheta_{1}^{\prime}(\tau)^{4}=Q_{1}^{\prime \prime \prime}(0 \mid \tau) Q_{2}^{\prime}(0 \mid \tau)-Q_{1}^{\prime}(0 \mid \tau) Q_{2}^{\prime \prime \prime}(0 \mid \tau) .
$$


From (7-2), it is easily seen that

$$
\begin{aligned}
& Q_{1}^{\prime}(0 \mid \tau)=4 q^{3 / 4} \sum_{n=-\infty}^{\infty}(-1)^{n}(3 n+2) q^{\left(3 n^{2}+4 n\right) / 4}, \\
& Q_{1}^{\prime \prime \prime}(0 \mid \tau)=-16 q^{3 / 4} \sum_{n=-\infty}^{\infty}(-1)^{n}(3 n+2)^{3} q^{\left(3 n^{2}+4 n\right) / 4}, \\
& Q_{2}^{\prime}(0 \mid \tau)=-4 q^{3 / 4} \sum_{n=-\infty}^{\infty}(3 n+2) q^{\left(3 n^{2}+4 n\right) / 4}, \\
& Q_{1}^{\prime \prime \prime}(0 \mid \tau)=16 q^{3 / 4} \sum_{n=-\infty}^{\infty}(3 n+2)^{3} q^{\left(3 n^{2}+4 n\right) / 4} .
\end{aligned}
$$

Substituting the four equations above and $\vartheta_{1}^{\prime}(\tau)=2 \eta^{3}(\tau)$ into (8-1) and simplifying, we arrive at Theorem 1.10.

Now we begin to prove Corollary 1.11 using Theorem 1.10. If we write

$$
(q ; q)_{\infty}^{10}=\sum_{n=0}^{\infty} b(n) q^{n}
$$

then by equating coefficients of $q^{n}$ for $n \geq 1$ on both sides of 1.10 , we deduce that

$$
b(n)=\frac{1}{4} \sum_{\substack{u, v=-\infty \\ 3 u^{2}+3 v^{2}+4 u+4 v+1=4 n}}^{\infty}\left((-1)^{u}-(-1)^{v}\right)(3 u+2)(3 v+2)^{3} .
$$

If $n \equiv 6(\bmod 11)$, then $3 u^{2}+3 v^{2}+4 u+4 v \equiv 1(\bmod 11)$. By inspecting all cases modulo 11 , we find that both $u, v \equiv 3(\bmod 11)$. It follows from $(8-3)$ that

$$
b(11 n+6) \equiv 0\left(\bmod 11^{4}\right) .
$$

Now from (8-2), we have

$$
\sum_{n=0}^{\infty} p(n) q^{n}=\frac{1}{(q ; q)_{\infty}}=\frac{(q ; q)_{\infty}^{10}}{(q ; q)_{\infty}^{11}} \equiv \frac{(q ; q)_{\infty}^{10}}{\left(q^{11} ; q^{11}\right)_{\infty}}=\frac{\sum_{n=0}^{\infty} b(n) q^{n}}{\left(q^{11} ; q^{11}\right)_{\infty}}(\bmod 11)
$$

Extracting those terms with indices of the form $11 n+6$ and employing (8-4), we conclude that

$$
\sum_{n=0}^{\infty} p(11 n+6) q^{11 n+6} \equiv \frac{\sum_{n=0}^{\infty} b(11 n+6) q^{11 n+6}}{\left(q^{11} ; q^{11}\right)_{\infty}} \equiv 0(\bmod 11) .
$$

Thus we have $p(11 n+6) \equiv 0(\bmod 11)$. This proves Corollary 1.11 . 
There are several different series representations of $\eta^{10}(\tau)$ in the literature.

Winquist [1969] derived an important identity, now known as the Winquist identity, which he then used to get the following identity for $\eta^{10}(\tau)$ :

$$
\begin{aligned}
48(q ; q)_{\infty}^{10}=\sum_{m, n=-\infty}^{\infty}(-1)^{m+n}(6 m+ & 3)(6 n+1) \\
& \times\left((6 m+3)^{2}-(6 n+1)^{2}\right) q^{\left(3 m^{2}+3 n^{2}+3 m+n\right) / 2} .
\end{aligned}
$$

Winquist then used this identity to give a simple proof of Ramanujan's partition congruence $p(11 n+6) \equiv 0(\bmod 11)$.

Berndt, Chan, Liu, and Yesilyurt [2004] used two results from Ramanujan's notebooks, and Liu [2005] used the theory of elliptic functions to prove

$$
\begin{aligned}
32(q ; q)_{\infty}^{10}=\sum_{m, n=-\infty}^{\infty}(-1)^{m+n}(2 m+ & 1)(2 n+1) \\
& \times\left(9(2 m+1)^{2}-(2 n+1)^{2}\right) q^{\left(9 m^{2}+n^{2}+9 m+n\right) / 6},
\end{aligned}
$$

which leads to a short proof of Ramanujan's congruence $p(11 n+6) \equiv 0(\bmod 11)$.

By using some Lambert series expansions for infinite products, Chan [2005] established that

$3(q ; q)_{\infty}^{10}=\sum_{m, n=-\infty}^{\infty}(3 m+1)(3 n+1)\left(4(3 m+1)^{2}-(3 n+1)^{2}\right) q^{3 m^{2}+2 m+\left(3 n^{2}+2 n\right) / 4}$.

Chu $[2005 ; 2007]$ used the method of difference equations to prove that

$$
3(q ; q)_{\infty}^{10}=\sum_{m, n=-\infty}^{\infty}(3 m+1)(6 n+1)\left(4(3 m+1)^{2}-(6 n+1)^{2}\right) q^{3 m^{2}+3 n^{2}+2 m+n},
$$

and then derived a proof of $p(11 n+6) \equiv 0(\bmod 11)$.

Chan, Cooper, and Toh [2007] provided the following formula by using a theta function identity:

$$
6(q ; q)_{\infty}^{10}=\sum_{m, n=-\infty}^{\infty}(6 m+1)(6 n+4)\left((6 m+1)^{2}-(6 n+4)^{2}\right) q^{3 m^{2}+m+3 n^{2}+4 n+1} .
$$

\section{Acknowledgment}

The author is grateful to the referee for many helpful suggestions.

\section{References}

[Andrews 1984] G. E. Andrews, "Hecke modular forms and the Kac-Peterson identities", Trans. Amer. Math. Soc. 283:2 (1984), 451-458. MR 85e:11031 Zbl 0545.10016

[Andrews 1986] G. E. Andrews, "The fifth and seventh order mock theta functions", Trans. Amer. Math. Soc. 293:1 (1986), 113-134. MR 87f:33011 Zbl 0593.10018 
[Andrews et al. 1999] G. E. Andrews, R. Askey, and R. Roy, Special functions, Encyclopedia of Mathematics and its Applications 71, Cambridge University Press, 1999. MR 2000g:33001 Zbl 0920.33001

[Berndt 1991] B. C. Berndt, Ramanujan's notebooks, III, Springer, New York, 1991. MR 92j:01069 Zbl 0733.11001

[Berndt 2006] B. C. Berndt, Number theory in the spirit of Ramanujan, Student Mathematical Library 34, American Mathematical Society, Providence, RI, 2006. MR 2007f:11001 Zbl 1117.11001

[Berndt et al. 2004] B. C. Berndt, S. H. Chan, Z.-G. Liu, and H. Yesilyurt, "A new identity for $(q ; q)_{\infty}^{10}$ with an application to Ramanujan's partition congruence modulo 11", Q. J. Math. 55:1 (2004), 13-30. MR 2004k:11166 Zbl 1060.11063

[Chan 2005] S. H. Chan, "Generalized Lambert series identities", Proc. London Math. Soc. (3) 91:3 (2005), 598-622. MR 2006i:33020 Zbl 1089.33012

[Chan et al. 2007] H. H. Chan, S. Cooper, and P. C. Toh, "Ramanujan's Eisenstein series and powers of Dedekind's eta-function", J. Lond. Math. Soc. (2) 75:1 (2007), 225-242. MR 2008c:11064 Zbl 1120.11021

[Chu 2005] W. Chu, "Theta function identities and Ramanujan's congruences on the partition function”, Q. J. Math. 56:4 (2005), 491-506. MR 2006j:11142 Zbl 1116.11086

[Chu 2007] W. Chu, "Quintuple products and Ramanujan's partition congruence $p(11 n+6) \equiv$ $0(\bmod 11) "$, Acta Arith. 127:4 (2007), 403-409. MR 2008d:11119 Zbl 1116.05009

[Enneper 1890] A. Enneper, Elliptische Functionen: Theorie und Geschichte, 2nd ed., Halle a.S., L. Nebert, 1890.

[Ewell 1982] J. A. Ewell, "Completion of a Gaussian derivation", Proc. Amer. Math. Soc. 84:2 (1982), 311-314. MR 83a:10084 Zbl 0484.05010

[Ewell 1995] J. A. Ewell, "Arithmetical consequences of a sextuple product identity", Rocky Mountain J. Math. 25:4 (1995), 1287-1293. MR 97e:11129 Zbl 0853.11029

[Hardy and Wright 1979] G. H. Hardy and E. M. Wright, An introduction to the theory of numbers, 5th ed., Oxford University Press, 1979. MR 81i:10002 Zbl 0423.10001

[Hecke 1959] E. Hecke, Mathematische Werke, Vandenhoeck \& Ruprecht, Göttingen, 1959. MR 21 \#3303 Zbl 0092.00102

[Hirschhorn 1983] M. D. Hirschhorn, “A simple proof of an identity of Ramanujan”, J. Austral. Math. Soc. Ser. A 34:1 (1983), 31-35. MR 84h:10067 Zbl 0501.10012

[Kac and Peterson 1980] V. G. Kac and D. H. Peterson, "Affine Lie algebras and Hecke modular forms", Bull. Amer. Math. Soc. (N.S.) 3:3 (1980), 1057-1061. MR 82b:10028 Zbl 0457.17007

[Klein and Fricke 1890] F. Klein and R. Fricke, Vorlesungen über die Theorie der elliptische Modulfunktionen, II, Teubner, Leipzig, 1890.

[Kongsiriwong and Liu 2003] S. Kongsiriwong and Z.-G. Liu, "Uniform proofs of $q$-series-product identities", Results Math. 44:3-4 (2003), 312-339. MR 2004j:33020 Zbl 1053.11015

[Liu 2002] Z.-G. Liu, "An expansion formula for $q$-series and applications”, Ramanujan J. 6:4 (2002), 429-447. MR 2006b:33031 Zbl 1044.05012

[Liu 2005] Z.-G. Liu, "A theta function identity and its implications", Trans. Amer. Math. Soc. 357:2 (2005), 825-835. MR 2005m:33036 Zbl 1063.11011

[Liu 2007] Z.-G. Liu, "An addition formula for the Jacobian theta function and its applications", Adv. Math. 212:1 (2007), 389-406. MR 2008c:11065 Zbl 05156392

[Rogers 1894] L. J. Rogers, "Second memoir on the expansion of certain infinite products", Proc. London Math. Soc. 25 (1894), 318-343. 
[Schoeneberg 1953] B. Schoeneberg, "Über den Zusammenhang der Eisensteinschen Reihen und Thetareihen mit der Diskriminante der elliptischen Funktionen", Math. Ann. 126 (1953), 177-184. MR 15,105b Zbl 0053.05403

[Shen 1994] L.-C. Shen, "On the additive formulae of the theta functions and a collection of Lambert series pertaining to the modular equations of degree 5", Trans. Amer. Math. Soc. 345:1 (1994), 323345. MR 95a:33038 Zbl 0808.33014

[Shen 1995] L.-C. Shen, "On some modular equations of degree 5", Proc. Amer. Math. Soc. 123:5 (1995), 1521-1526. MR 95f:33017 Zbl 0826.33011

[Shen 1999] L.-C. Shen, "On the products of three theta functions", Ramanujan J. 3:4 (1999), 343357. MR 2000k:33038 Zbl 1011.11035

[Winquist 1969] L. Winquist, "An elementary proof of $p(11 m+6) \equiv 0(\bmod 11)$ ", J. Combinatorial Theory 6 (1969), 56-59. MR 38 \#4434 Zbl 0241.05006

Received March 27, 2008. Revised November 28, 2008.

\author{
ZHI-GUO LIU \\ DEPARTMENT OF MATHEMATICS \\ EAST CHINA NORMAL UNIVERSITY \\ 500 DONGCHUAN ROAD \\ SHANGHAI 200241 \\ CHINA \\ zgliu@math.ecnu.edu.cn \\ liuzg@hotmail.com
}

